\title{
Psicodinâmica do adolescente envolvido com drogas
}

\author{
Felix Kessler* \\ Lisia von Diemen** \\ Ana Carolina Seganfredo** \\ Iversom Brandão** \\ Patrícia de Saibro* \\ Bruno Scheidt* \\ Rodrigo Grillo*** \\ Sérgio de Paula Ramos ${ }^{\star * * *}$
}

"sobre o uso de drogas... trata-se de atos que visam ao alívio da dor ou apenas ao desejo do prazer?"

Thomas de Quincey, 1821. Confissões de um comedor de ópio.

\section{INTRODUÇÃO}

O consumo de tabaco por adolescentes escolarizados dobrou nos últimos quinze anos. O uso de maconha quadruplicou e o uso de cocaína multiplicou-se por dez, em Porto Alegre $^{2}$. Esses dados tornam o estudo sobre o uso de drogas, entre adolescentes, extremamente relevante. Além da alta prevalência, a gravidade dos problemas associados a esse consumo é preocupante. Da mesma forma, o primeiro levantamento domiciliar sobre uso de drogas realizado no Brasil $^{3}$ mostra uma prevalência de

\footnotetext{
* Médico psiquiatra, mestrando em Psiquiatria Universidade Federal do Rio Grande do Sul.

** Médico psiquiatra, Hospital de Clínicas de Porto Alegre.

${ }^{* * *}$ Médico psiquiatra, Hospital Presidente Vargas.

${ }^{* * *}$ Médico psiquiatra, Hospital Mãe de Deus.

${ }^{* * * *}$ Médico psiquiatra e psicanalista, Hospital Mãe de Deus.
}

dependência de álcool, em adolescentes de 12 a 17 anos, de $5.2 \%$. Essa mesma pesquisa aponta os maiores índices de dependência de tabaco e maconha, assim como o uso na vida de maconha e cocaína, para a região sul do Brasil.

A gravidade desses números fica potencializada pelo alto índice de comorbidade psiquiátrica relacionado ao uso de drogas nessa faixa etária. O Methodos for the Epidemiology of Child and Adolescent Mental Disorder (MECA $)^{4}$ encontrou um risco 20 vezes maior de haver algum Transtorno de Conduta (incluindo personalidade anti-social, déficit de atenção e transtorno opositivo desafiante) entre os adolescentes com abuso ou dependência atual de álcool, maconha ou outra droga ilícita. Dos adolescentes dependentes químicos, $76 \%$ apresentaram outro diagnóstico, comparado com $24.5 \%$ dos que não utilizavam substâncias psicoativas. Clark e colaboradores ${ }^{5}$ encontraram que adolescentes com dependência de álcool têm 7.1 vezes mais chance de ter Transtorno de Conduta, 2 vezes mais Transtorno Opositivo Desafiante, 2.8 vezes mais Transtorno de Déficit de Atenção e Hiperatividade e 3.1 vezes

Recebido em 24/01/2003. Revisado em 11/03/2003. Aprovado em 18/03/2003. 
mais Depressão do que os controles. Aqueles que buscavam tratamento tinham maior chance de ter algum outro transtorno psiquiátrico associado, mais estressores psicossociais e pior funcionamento social.

As conseqüências na vida dos adolescentes decorrentes do uso de drogas são inúmeras e muito graves. O suicídio na adolescência, por exemplo, apresenta uma forte relação com o uso de substâncias psicoativas ${ }^{6}$.

A importância desses fatos contrasta com a carência de estudos controlados sobre a eficácia das técnicas terapêuticas utilizadas nesta faixa etária, bem como de seus respectivos suportes teóricos. Por isso, $\mathrm{Kaminer}^{7}$ destaca a tendência dos profissionais dessa área de aplicarem as mesmas técnicas utilizadas com os adultos.

Um dos objetivos desta revisão é descrever os principais achados de pesquisa na área de adolescência e drogas, principalmente no que concerne aos fatores de risco e proteção para o seu uso. Pretende-se também analisar algumas das principais teorias psicanalíticas relacionadas à dependência química na adolescência e discutir a sua utilidade clínica no contexto das abordagens terapêuticas. Não se visa a avaliar detalhadamente as técnicas utilizadas no tratamento dos adolescentes dependentes químicos, mas tecer algumas considerações sobre esse tema para tornar mais clara a relevância da psicodinâmica.

É importante esclarecer que as teorias a serem discutidas a seguir, de forma alguma, invalidam ou excluem as novas descobertas das neurociências na área da dependência química, tampouco questionam os resultados positivos obtidos por outras formas de intervenção (técnicas cognitivo-comportamentais, entrevista motivacional, modelo dos 12 passos, aconselhamento, grupos, e outros tratamentos psicosociais). Desse modo, espera-se contribuir para um melhor entendimento e tratamento desses pacientes, privilegiando-se o paradigma psicodinâmico.

\section{CONTEXTUALIZAÇÃO DAS \\ DROGAS NA ADOLESCÊNCIA}

\section{Evidências clínicas:}

Fatores de risco e proteção para uso de drogas na adolescência:

Diversos estudos foram realizados na tentativa de determinar fatores de risco e de proteção com relação ao uso de drogas na adoles- cência. Sussman e colaboradores ${ }^{8}$ evidencia- $^{-}$ ram, como fatores de risco, ser do sexo masculino, fumar, ter inabilidade de lidar com a raiva e apresentar depressão. Outro achado desse estudo foi que o adolescente que não mora com um dos pais tem mais risco de usar drogas. Em revisão realizada por Simkin ${ }^{9}$ sobre o tema, encontrou-se como fatores de risco: 1) cultural e social: permissividade social, disponibilidade de droga, extrema privação econômica e morar em favela 2) interpessoal: a) na infância - família com conduta álcool e droga relacionadas, pobre e inconsistente manejo familiar, personalidade dos pais e abuso físico b) na adolescência - conflitos familiares e ou sexual, eventos estressantes (como mudança de casa e escola), rejeição dos seus pares na escola ou outros contextos, associação com amigos usuários. 3) Psicocomportamental: precoce e persistente problema de conduta, fracasso escolar, vínculo frágil com a escola, comprometimento ocupacional, personalidade antisocial, psicopatologia (Transtorno de Déficit de Atenção e Hiperatividade, depressão e transtorno de conduta, ou ansiedade nas mulheres), atitudes favoráveis para drogas, inabilidade de esperar gratificação 4) Biogenético: genealogia positiva para dependência química e vulnerabilidade psicofisiológica ao efeito de drogas. Em contrapartida, o estudo descreve alguns fatores de proteção: ambiente estável, alto grau de motivação, forte vínculo pais-criança, supervisão parental e disciplina consistentes, ligação com instituições pró-sociais e associação com amigos não usuários. Outro achado relatado foi que o tratamento precoce do THDA reduz em $85 \%$ o envolvimento com drogas.

Brown ${ }^{10}$ enfatiza que o exercício de colocação de limites por parte dos pais, o monitoramento familiar e ter uma refeição diária junto com os filhos funciona também como fator de proteção. Além desses, afirma que a religiosidade praticada e o trabalho comunitário seriam fatores de proteção familiar.

Miles $^{11}$, ao estudar a relação do uso de drogas na adolescência com características dos pais, percebeu que apenas um terço deles apareceram para a entrevista. O número de casais (pais) que tinham relacionamento informal $(61 \%$ dos dependentes químicos versus $7 \%$ dos não dependentes), problemas com o álcool, drogas ilícitas e transtorno de personalidade antisocial era superior aos dos controles. As mães apresentavam maior prevalência de tabagismo, alcoolismo e uso de cocaína. Nessa mesma linha, Bensley ${ }^{12}$ acrescenta que adolescentes com história de abuso físico ou sexual na infân- 
cia têm 2.8 vezes mais chance de uso leve ou moderado de drogas na adolescência e 3.4 de uso pesado. Crianças que sofreram abuso físico ou sexual na infância têm 12.2 vezes mais chance de experimentar maconha ou usar álcool antes dos 10 anos. Ademais, quanto mais precoce o abuso do álcool ou drogas, mais estaria relacionado com abuso físico ou sexual na infância.

Newcomb ${ }^{13}$ demonstrou ainda que, quanto maior o número de fatores de proteção, menor será o consumo de drogas pelos adolescentes, e, caso inverso, quanto maior o número de fatores de risco, maior a prevalência de consumo.

\section{Estudos Prospectivos}

Entre tantos estudos que envolveram a busca da etiologia da dependência química, destacaram-se aqueles que partiram de amostras de crianças e as estudaram por décadas, na esperança de identificar diferenças (e semeIhanças) entre aquelas que foram desenvolvendo alcoolismo e as que não o foram ${ }^{14}$.

Dentre os estudos prospectivos, dois deles serão privilegiados nesta revisão, devido às suas qualidades metodológicas. McCord e McCord $^{15}$ foram capazes de localizar, durante quase 30 anos, 255 dos 325 meninos estudados. Esses "meninos", ao final do estudo, estavam com idades entre 30 e 35 anos. Eles demonstraram que os futuros alcoolistas foram, na infância, mais autoconfiantes, menos perturbados por medos normais, mais agressivos, hiperativos e heterossexuais que os que não desenvolveram alcoolismo.

Talvez o mais interessante e discutido dos estudos prospectivos seja 0 de Vaillant ${ }^{16}$, que escreveu o livro A História Natural do Alcoolismo, por possuir amostra e seguimento maiores. Este autor e seus colaboradores acompanharam sua amostra de 660 indivíduos, procedentes de duas subamostras (de 204 e 456 meninos, respectivamente) por mais de 50 anos. Após todo este tempo, ele dispõe de dados de 559 "meninos".

Com relação à teoria da oralidade, Vaillant" descreve que "não há mais oralidade entre crianças que serão abusadoras de álcool, comparadas com as demais. Entretanto, existe mais oralidade entre alcoolistas que nos grupos controles, ou seja, a oralidade vem com o alcoolismo em vez de precedê-lo". Vaillant ${ }^{16,18}$ também estudou o impacto da figura da mãe nos meninos de sua amostra. Concluiu que "mães que proporcionaram cuidados inadequados não aumentaram a chance de ter filhos alcoolistas, $e$, mais, que mães que proveram relações calorosas não tiveram menos alcoolismo do que as demais na prole. Tais achados contrastam com os relativos à figura paterna, pois claramente uma relação calorosa com o pai foi capaz de gerar menos alcoolismo, e, inversamente, foi marcante a prevalência de abuso alcoólico entre aqueles que tiveram má relação com o pai". Para este autor, seria maior o fator protetor de boas relações na infância do que etiogênica a presença de fatos traumáticos. A hereditariedade, a etnicidade (ser latino) e a presença de comportamentos anti-sociais na infância foram os fatores mais associados ao futuro alcoolista, na amostra estudada por Vaillant.

\section{Psicodinâmica do adolescente envolvido com drogas:}

Sabe-se que, ao passar para a adolescência, o jovem experimenta uma mudança tanto fisiológica quanto psicológica. Ao lado das modificações em seu corpo, também surgem transformações nas suas percepções em relação a si próprio e aos outros. Ele passa, então, por um período de maior fragilidade egóica. O resultado é uma volta narcísica para o seu mundo interno, com questionamentos sobre os pais, as instituições e a sociedade. Esta volta narcísica provoca uma série de ansiedades naturais do período, como as da identidade pessoal, as depressivas, pela perda da identidade infantil, e até paranóides, devido à luta interna que passa a travar em busca desse novo conhecimento. Concomitantemente, há a formação de novos grupos, mas é também um período de isolamento, em que o jovem busca compreender as mudanças pelas quais está passando.

De forma ampla, o entendimento psicodinâmico dos adolescentes tem sido um desafio para os profissionais da saúde mental. Tornase ainda mais complexa a compreensão daqueles que utilizam substâncias psicoativas. São escassos os estudos nessa área, e poucos autores arriscaram-se a aprofundar esse tema. No Brasil, por exemplo, a Revista Brasileira de Psicanálise, nos últimos 30 anos, nada publicou sobre a matéria.

Várias teorias psicodinâmicas sobre a gênese desta condição já foram desenvolvidas: teoria das gratificações narcísicas, teoria da oralidade, teoria das relações maníacas e teoria das perversões. Rosenfeld ${ }^{19}$ concluiu que havia um consenso entre a maioria dos autores da época que em ambos (uso de drogas e alcoolismo) havia "uma importância dos aspec- 
tos orais, mania, depressão, impulsos destrutivos e auto-destrutivos e perversão, tais como a homossexualidade e o sadomasoquismo". No entanto, desde então, os autores centraram-se em outros pontos que pensam ser mais relevantes. Para este trabalho foram selecionados os trabalhos daqueles autores de reconhecida reputação e com maior número de publicações na área da dependência química. Algumas dessas teorias serão comentadas ou complementadas com comentários ou vinhetas clínicas dos autores deste artigo.

Atualmente, a maioria dos autores concorda que o envolvimento com substâncias psicoativas implica uma relação narcisística. Clark ${ }^{20}$, mesmo em 1919, já sublinhava a importância das regressões profundas no alcoolismo, tais como as identificações primárias com a mãe, combinadas ao intenso auto-amor (narcisismo); Kielholz ${ }^{21}$ incluiu o alcoolismo como uma neurose narcísica. Rosenfeld ${ }^{21}$ refere que os dependentes seriam portadores de inveja primária do seio materno, o que levou a paciente que ilustra seu trabalho a precocemente preterir o seio em favor de seu próprio polegar. A droga, nesta perspectiva, seria um substituto deste polegar.

Apesar de inúmeros autores, além dos já citados, no início do século vinte, terem elaborado algumas teorias sobre a dependência química de adultos, a maioria dos artigos que se referem a adolescentes sugiram a partir da década de 70. Ainda assim, é válido ressaltar que algumas das teorias abaixo relatadas também foram desenvolvidas na análise de casos de adultos.

Kohut $^{22,23}$ investigou jovens dependentes e concluiu que eles carecem de um objeto bom interiorizado. As funções paterna e materna encontram-se comprometidas. A personalidade encontra-se privada de coesão, como se faltasse a imagem idealizada do pai e a empatia da mãe. Nesse contexto, as drogas transformariam a realidade ansiogênica em neutra, reforçando nos usuários a sua onipotência. "É o triunfo da negação". Segundo ele, a função da terapia seria proporcionar uma gradual identificação e introjeção de elementos bons pelo paciente, com o estabelecimento de uma forte aliança terapêutica.

Entretanto, o vínculo inicial com os dependentes químicos é extremamente frágil e, devido a essa negação e onipotência, é importante cativá-los, evitando confrontações ou posturas que possam ser interpretadas como autoritárias ou preconceituosas.

Krystal ${ }^{24}$ também fez importantes contribui- de psíquica do dependente de drogas é dominada por uma forte experiência de ambivalência na relação com a imagem da mãe, posteriormente estendida à droga e a outras pessoas do seu círculo de relações, incluindo o terapeuta. Ele diz: "ao mesmo tempo que o adicto clama pelo amor objetal da mãe, ele o despreza" e relaciona isso ao fim do efeito da droga, uma vez que, além da garantia do prazer (aproximação da mãe), o usuário também tem a garantia do pós-efeito (distanciamento). Não deixa de ser um controle onipotente do objeto. Ele ainda acrescenta que muitos usuários de drogas apresentam uma certa alexitimia (incapacidade de expressar os seus sentimentos), como se houvesse uma economia dos afetos e de sua não representação.

Uma completa união com o objeto parece ser ameaçadora, uma vez que o caráter ambivalente da relação significa que ele está contaminado com intenso ódio, inveja e medo de ser ferido, uma visão compartilhada com outros autores como Kernberg ${ }^{25}$. Como conseqüência da ambivalência, a criança não é capaz de introjetar a imagem da mãe, o que leva a uma falta das funções de auto-cuidado, comum nos adictos. Soma-se a isso um sentimento de insegurança e dependência.

Khantzian ${ }^{26}$, um dos autores mais produtivos dessa área, postula que indivíduos dependentes de drogas apresentam uma predisposição ao uso e a se tornarem dependentes, principalmente devido a um severo prejuízo do ego e distúrbios do senso do self, envolvendo dificuldades com instintos, affect defense, autocuidados, dependência e necessidade de satisfação. As enormes e persistentes dificuldades dos dependentes de heroína chamaram, desde muito cedo, a atenção do autor, principalmente no que diz respeito aos sentimentos e impulsos associados à agressão. A maneira drástica como a heroína aliviava os sentimentos disfóricos de raiva e desassossego foi um ponto comum encontrado nas observações clínicas do pesquisador, levando-o a acreditar que o uso da droga poderia ser visto como uma maneira de auto-medicação, sua hipótese mais conhecida. Segundo essa teoria, os efeitos psicoativos específicos de cada droga interagem com os transtornos psiquiátricos e estados afetivos dolorosos.

Ilustra esse ponto o relato de um paciente, jovem de dezenove anos, com uma história de cinco anos de abuso de cocaína, que relatou, durante a entrevista inicial, uma persistente sensação de dificuldade de socialização, baixa auto-estima, iniciadas no início da adolescên- 
cia. Contou que com a cocaína passara a sentir-se mais "solto", com mais energia para fazer suas atividades diárias e menos preocupado com a opinião ou julgamento dos outros em relação a si.

Olievenstein ${ }^{27}$ chama a atenção para o problema da falta. Para esses autores, talvez mais importante do que o prazer narcísico propiciado pelas drogas, estaria um sentimento de falta, geralmente aliviado por elas. As mães não suficientemente boas (Winnicott ${ }^{27}$ ) gerariam um estado de crônica falta. Uma falta oceânica e jamais saciável. Desta ótica, o depender de drogas seria o resultado do deslocamento deste sentimento de falta para uma "coisa", com a notória vantagem de esta ser alcançável em qualquer esquina do mundo ${ }^{14}$.

David Rosenfeld ${ }^{28}$, concordando com Khantzian, observa diversas estruturas psicopatológicas comuns na drogadição, com sua própria dinâmica inconsciente e psicogênese infantil, em que cada indivíduo procura a droga por um determinado motivo. Certos pacientes não têm noção de perigo, pelo comprometimento dos processos de introjeção dos objetos parentais e sentem o seu mundo interno esvaziado e sem vida. Constantes condutas de risco podem ser entendidas, então, como fruto da necessidade de sentirem-se vivos.

Um adolescente de dezessete anos, usuário de múltiplas drogas desde os seus 11 anos, referia uma história de abandono pelos pais na infância, tendo sido criado pela avó materna, junto com seus três irmãos. Desde cedo, envolveu-se em furtos e roubos. O jovem descrevia sentimentos crônicos de vazio interno e descontrole de impulsos. Contou que obtinha prazer nas situações de alto risco de vida. De forma impressionante, após uma overdose de cocaína, dizia sentir-se mais vivo.

Outra autora que fala sobre a relação da droga com os estados afetivos é Joyce Mc Dougall ${ }^{29}$. Ela coloca o comportamento adictivo como uma solução à intolerância afetiva. O objeto de adição seria experimentado como essencialmente bom, um objeto idealizado, com uma promessa de prazer e capaz de resolver magicamente as angústias e os sentimentos de morte interna. A solução adictiva teria origem principalmente na relação mãe-bebê, quando a mãe sente-se fusionada ao bebê e cria uma relação de dependência do bebê à sua presença. Isso dificultaria que a criança constituísse em seu mundo interno as representações maternas e, mais tarde, paterna cuidadoras, capazes de conter e manejar seus estados de sofrimento psíquico. A falta de objetos internos de identificação para aliviar por si mesmo seus estados de tensão psíquica ocasionaria mais tarde uma busca no mundo externo de algo que substituísse a mãe, como a droga. O objeto adictivo seria então um objeto transitório, como postula a autora, e não transicional, uma vez que resolveria momentaneamente a tensão psíquica através de solução somática e não psicológica.

Quanto às questões técnicas, diversos autores descrevem que a contratransferência com o dependente químico é semelhante àquela que se experimenta com pacientes psicóticos, provocando reações de intensa frustração, ódio ou desesperança, devido às freqüentes recaídas. Alguns pacientes podem ter a necessidade de serem hospitalizados várias vezes antes de alcançarem a abstinência.

Imhof ${ }^{30}$ refere que esses pacientes fazem com que o psicanalista,muitas vezes, cumpra uma função de um objeto inanimado, ou seja, uma droga. "O perigo que o psicanalista corre é começar a funcionar como um objeto não humano, respondendo ao que foi transferido". É muito sutil a forma com que o paciente vai transformando o analista em um objeto inanimado, a ser usado nos momentos de necessidade. Ele explica que é importante que o terapeuta perceba esse tipo de relação para poder revertê-la.

Uma moça de 22 anos, com uma história de 7 anos de uso de maconha e cocaína, costumava procurar o seu terapeuta somente após o uso de drogas. Solicitava sessões extras e, quando isso não era possível, propunha um encontro rápido, mesmo que fosse nos intervalos de 10 minutos entre as sessões, para poder desabafar, e no dia da sessão não comparecia.

Nesses casos, o terapeuta deve cuidar para não se comprometer com o superego dos pais, tampouco assumir uma posição maternal de extremo zelo e preocupação ${ }^{31}$. Ele deve estar ciente de que as interpretações podem ser desvalorizadas, o que, freqüentemente, pode despertar contra-atitudes negativas do terapeuta. Os desafios e agressões impõem ao terapeuta uma atitude de abstenção de toda contra-agressão.

\section{ALGUMAS CONSIDERAÇÕES TERAPÊUTICAS}

Historicamente, as abordagens psicoterapêuticas utilizadas com usuários de drogas têm sido um reflexo das modalidades mais proeminentes, em cada época particular, aplicadas para os outros transtornos mentais. A técnica 
psicanalítica clássica já foi indicada, há algumas décadas, como o tratamento de escolha para esses pacientes. Contudo, esse modelo terapêutico isoladamente demonstrou resultados desanimadores. Este fato tem sido atribuído à falta de ênfase no controle dos sintomas relacionados ao uso da droga e pelo foco mais dirigido aos aspectos psicodinâmicos, em detrimento dos aspectos biológicos e sociais. Ilustra essa dificuldade, como Ramos ${ }^{32}$ demonstrou, o ocorrido com Anna O., a primeira paciente da história da psicanálise. Breuer tratou-a, subestimando os efeitos do consumo de hidrato de cloral e morfina praticados pela paciente. Com isso, duas semanas após ter dado-lhe alta, a paciente precisou ser hospitalizada novamente.

A clássica neutralidade analítica e as interpretações tendem a ser geradores de ansiedade, desencadeando recaídas ${ }^{33}$. Entretanto, um número de diferentes intervenções de "orientação psicodinâmica" já foi desenvolvido, tais como intervenção em crise, terapia suportiva e psicoterapia expressiva, e algumas delas já foram testadas em dependentes químicos com resultados semelhantes aos de outras técnicas cognitivo-comportamentais, psicosociais, grupos de auto-ajuda e em forma de aconselhamento $^{34,35}$. Outros estudos mostram ainda que, a longo prazo, a psicoterapia psicodinâmica provou ser mais efetiva do que o aconselhamento $^{35}$.

Devido às inúmeras razões relacionadas ao melhor entendimento do paciente e ao vínculo com o terapeuta, a perspectiva psicodinâmica torna-se uma ferramenta muito útil na conceitualização dos dependentes químicos, ajudando na formulação das estratégias comportamentais efetivas e no aprofundamento da qualidade da recuperação desses pacientes. Sabe-se também que os distúrbios emocionais e interpessoais, abordados nas terapias psicodinâmicas, são importantes precipitantes de "fissuras" e recaídas. O manejo desses aspectos é extremamente relevante para a prevenção destas recaídas ${ }^{36}$.

Entretanto, a maioria dos estudiosos dessa área alega que os dependentes químicos devem estar estabilizados em relação à sua abstinência das drogas ${ }^{37}$ ou, pelo menos, no estágio de mudança de ação ${ }^{38}$, para poderem beneficiar-se das abordagens psicodinâmicas. Além disso, esses pacientes deverão ter uma percepção do sofrimento intrapsíquico, um desejo pelo auto-conhecimento - mais que puramente a remoção dos sintomas - e uma habilidade de atribuir parte do seu sofrimento aos problemas internos ${ }^{29}$
A eficácia da psicoterapia dinâmica para adolescentes dependentes químicos ainda é um campo a ser melhor explorado, em relação aos adultos ${ }^{39}$

Um estudo interessante, realizado com dependentes químicos, avaliou o desempenho dos terapeutas e suas variações, demonstrando que terapeutas mais interessados e capazes de uma melhor aliança terapêutica, bem como os que conseguiam prover uma relação mais calorosa tinham melhores resultados ${ }^{40}$. A confidencialidade também parece ser algo fundamental, que necessita ser discutida com os pacientes adolescentes, uma vez que $25 \%$ dos pacientes relatam que abandonariam o tratamento caso soubessem que o sigilo seria rompido ${ }^{41}$.

A escolha da abordagem terapêutica está intrinsecamente ligada ao tipo de paciente e à fase (ou estágio de mudança) do tratamento em que o paciente se encontra. Sabe-se que qualquer tratamento, em dependência química, é melhor que nenhum tratamento e que 10 anos após o Projeto Match $^{42}$, que comparou três tipos de tratamentos em um grande número de alcoolistas (após 1 ano, os pacientes bebiam $75 \%$ menos dias e $80 \%$ menos quantidade, mas apenas $35 \%$ estavam abstêmios), nenhum tipo de tratamento para dependentes químicos consegue alcançar a abstinência total em muito mais do que um terço dos pacientes, após um ano de tratamento.

Considerando o acima exposto, Wurmser ${ }^{43}$ propõe um tratamento seqüencial, no qual, em primeiro lugar, tentar-se-á fazer com que o paciente se desintoxique. Quando possível, em regime ambulatorial, mas sempre que necessário, com hospitalizações em unidades especializadas. Durante este primeiro momento, todos os esforços deverão ser engendrados para se fazer uma completa avaliação diagnóstica do paciente e de sua família, bem como, através da utilização de técnicas motivacionais, ajudar o paciente a aderir ao tratamento. É necessária uma abordagem mais suportiva, não confrontativa e mais diretiva por parte do terapeuta.

Utilizam-se também nessa fase, técnicas de grupo e de aconselhamento. Uma vez o paciente desintoxicado, diagnosticado e motivado, as terapias cognitivo-comportamentais já comprovaram sua eficácia na manutenção da abstinência, que deve ser monitorada pela feitura de screenings de drogas na urina duas vezes por semana. A maioria dos casos necessitará permanecer em tratamento por um ano ou mais dentro desse referencial terapêutico, até que reúna condições de estabilidade para um trabalho orientado psicanaliticamente. 
Se for constatada a presença de comorbidade, esta deve ser tratada da forma mais conveniente, associando-se farmacoterapia à psicoterapia. Da mesma forma, famílias disfuncionais deverão receber indicação de terapia de família. As terapias seqüenciais, ou as combinadas, no tratamento das dependências químicas, são vistas como uma estratégia terapêutica ${ }^{44}$.

\section{CONCLUSÃO}

Através dessa revisão, pôde-se constatar a relevância do estudo do envolvimento dos adolescentes com o uso de substâncias psicoativas, assim como a abrangência desse assunto, tanto pela compreensão teórica, quanto pela definição das abordagens terapêuticas.

Percebeu-se uma carência de trabalhos psicanalíticos nessa área, assim como de pesquisas com os adolescentes usuários de drogas, sendo que poucos autores arriscaram-se a tecer considerações terapêuticas e aprofundarse nesse tema.

Em relação às teorias psicanalíticas, o fato é que, sejam por atributos maternos, por características do próprio indivíduo (constitucionais ou não), ou sejam ainda por ambos, parece haver uma concordância, entre os autores revisados, de que existiria fundamentalmente uma relação narcísica objetal na qual a substância seria a fonte de prazer narcísico ${ }^{14}$.

É interessante notar que as contribuições dos psicanalistas citados encontram suporte nos estudos prospectivos sobre fatores de risco e proteção mencionados no início do artigo, estabelecendo uma relação clara entre a vivência psíquica dos cuidados parentais e a função da droga no contexto afetivo de cada paciente. As evidências clínicas também indicam a hipótese de que o comprometimento de aspectos da função paterna, que inclui o monitoramento e definição dos limites, pode ser um fator preponderante para o desencadeamento e manutenção da dependência química.

Pelo exposto neste trabalho, ainda ficou evidente que a tarefa de tratar um adolescente envolvido com drogas é complexa, e as diferentes escolas psicoterápicas têm apresentado resultados modestos, nenhuma delas sendo capaz de atender os diferentes problemas impostos, sugerindo-se tratamentos seqüenciais ou combinados.

Essa proposta é de difícil consecução e, muitas vezes, pode ser mais indicado um trabaIho com uma equipe de diferentes profissionais da área da saúde, que possa tratar esses pro- blemas em conjunto. Deve-se evitar oferecer ao paciente o que "sei fazer", dispondo-lhe o tipo de tratamento mais adequado naquele momento.

Assinala-se ser indicado, após um período sustentável de manutenção da abstinência e quando o paciente desejar e puder, a subseqüente psicoterapia de orientação analítica, ou mesmo psicanálise, para a elaboração da relação simbiotizada e dos aspectos narcísicos rumo a uma relação de objeto independente, mesmo que isso exija o encaminhamento para um profissional especializado nessa técnica.

\section{REFERÊNCIAS BIBLIOGRÁFICAS}

1. De Quincey T. Confissões de um comedor de ópio. Porto Alegre: Editora L\&P; 2001.

2. Ramos SP, Saibro P. Prevalência do consumo de drogas por adolescentes escolarizados na cidade de Porto Alegre. Comunicação pessoal; 2003

3. Carlini EA, Galduróz JE, Noto AR, Nappo SA. I levantamento domiciliar sobre uso de drogas no Brasil. Estudo envolvendo as 107 maiores cidades do país - 2001 . Secretaria Nacional Anti-Drogas (SENAD) e Centro Brasileiro de Informações sobre Drogas Psicotrópicas (CEBRID). São Paulo: Cromosste Gráfica e Editora Ltda; 2002.

4. Kandel DB, Johonson JG, Bird HR, Weissman MN, Goodman SH, Lahey BB, et al. Psychiatric comorbidity among adolescent with substance abuse disorders: findings from MECA study. J Am Acad Child Adolesc Psychiatry 1999 june;38 (6): 693-99.

5. Clark DB, Pollock N, Bukstein OG, Mezzich $A C$,Bromberger JT, Donovan JE. Gender and comorbid psychopathology in adolescents with alcohol dependence. J Am Acad Child Adolesc Psychiatry 1997 Sep; 36(9):1195-203.

6. Rowan AB. Adolescent substance abuse and suicide. Depress Anxiety 2001; 14(3):186-91.

7. Kaminer $Y$. Adolescent substance abuse treatment: Where do we go from here? Psychiatric Services 2001; 52 (2): 147-49.

8. Sussman S, Dent CW, Galaif ER. The correlates of substance abuse and dependence among adolescents at high risk for drug abuse. J Subst Abuse 1997; (9): 241-53.

9. Simkin DR. Adolescent substance use disorders and comorbidity. Pediatr Clin N Am 49 2002; (49): 463-77.

10. Brown RT. Risk factors for substance abuse in adolescents. Pediatr Clin N Am 2002; (49): 247-55.

11. Miles DR, Stallings MC, Young SE, et al. A family history and direct interview study of the familial aggregation of substance abuse: the adolescent substance abuse study. Drug Alcohol Depend 1998; (49): 105-14.

12. Bensley LS, Spieker SJ, Van Eenwyk J, Schoder J. Selfreported abuse history and adolescent problem behaviors. II. Alcohol and drug use. J Adolesc Health 1999 Mar; 24(3):173-80

13. Newcomb MD. Identifying high-risk youth: prevalence and patterns of adolescent drug abuse. In: Adolescent Drug Abuse: clinical assessment and therapeutic interventions. NIDA Res Monogr 1995; 156.

14. Ramos SP. A questão das drogas e a Psicanálise. TrabaIho para membro efetivo da Sociedade Psicanalítica de Porto Alegre; 1998.

15. McCord W. \& McCord J. Origins of alcoholism. Stanford: 
Stanford University Press; 1960.

16. Vaillant $\mathrm{G}$. The natural history of alcoholism. Cambridge: Harvard University Press; 1983.

17. Vaillant G. Natural history of male psychological health VIII: Antecedents of alcoholism and "orality". Amer $J$ Psychiatry 1980; (137):181-186.

18. Vaillant G. The natural history of alcoholism revisited. Cambridge: Harvard University Press; 1995.

19. Rosenfeld HA. Psicopatologia da toxicomania e do alcoolismo (revisão da literatura - 1964). In: Rosenfeld HA. Os estados psicóticos. Rio de Janeiro: Zahar; 1968.

20. Clark LP. A psychological study of some alcoholics. Psychoanal 1919; (6): 36-42.

21. Rosenfeld HA. Da toxicomania. In: Rosenfeld HA. Os estados psicóticos. Rio de Janeiro: Zahar, 1968.

22. Kohut $\mathrm{H}$. Thoughts on Narcissism and Narcissistic Rage. Psychoanalytic Study of the Child 1972; (27):360-400.

23. Kohut H. Self Deficits and Addiction. In: Psychodynamics of Drug Dependence. NIDA Res Monogr 1977; 12.

24. Krystal H. Self representation and the capacity for selfcare. Annual of Psychoanalysis 1978; (6): 209-46.

25. Kernberg, O. Borderline conditions and pathological narcissism. New York: Jason Aronson; 1975.

26. Khantzian EJ: The self-medication hypothesis of addictive disorders: Focus on heroin and cocaine dependence. Am J Psychiatry 1985; (142): 1259-1264.

27. Olievenstein C. A clínica do toxicômano. Porto Alegre: Ed. Artes Médicas; 1990.

28. Rosenfeld D. Nuevas teorías sobre drogadicción. Autismo y drogadicción. Revista de la Asociacion Psicoanalitica de Buenos Aires 1996; XVIII (2):347-64.

29. Mc Dougall J. Neonecesidades y Soluciones Adictivas. Piscoanalisis con Niños y Adolescentes 1998 Octubre; (11):62-78

30. Imhof J. Countertransferential and attitudinal considerations in the treatment of drug abuse and addiction. The international Journal of Addictions 1983; (18) 491-510.

31. Imhof J. Countertransference issues in alcoholism and drug addiction. Psychiatric Annals 1991; (21): 292-306.

32. Ramos, SP. Anna O.: a case of chemical dependence. History of psychology. In press 2003

33. Acuña G. A Psychodynamic Contribution to Addiction. Addiction. In Press 2003.

34. Barber, J. \& Crits-Christoph, P. Psychodynamic psychotherapies for psychiatric disorders (axis I). New York, Basic Book; 1995.

35. Crits-Christoph $\mathrm{P}$, Beebe KL, Connolly MB: Therapist effects in the treatment of drug dependence: Implications for conducting comparative treatment studies, in Psychotherapy and Counseling in the Treatment of Drug Abuse. NIDA Res Monogr 1990; 104.

36. Marlatt GA, Gordon JR. Prevenção de recaída. Porto Alegre: Artes Médicas 1993.

37. Carroll KM, Rounsaville BJ, Treece FH. A comparative trial of psychotherapies for ambulatory cocaine abusers: Relapse prevention and interpersonal psychotherapy. Am $\mathrm{J}$ of Drug Alcohol Abuse 1991;17(3): 229-247

38. Prochaska JO, di Clemente CC. Stages and processes of self-change of smoking: toward an integrative model of change. J Consult Clin Psychol 1983; (51): 390-395.

39. Woody GE, McLellan AT, Luborsky L, O'Brien CP, Blaine $J$, Fox S, et al. Severity of psychiatric symptoms as a predictor of benefits from psychotherapy: the Veterans Administration-Penn study. Am J Psychiatry 1984; (141): 1172-1177.

40. Luborsky L, McLellan T, Woody GE. Therapist success and its determinants. Arch Gen Psychiatry 1985; (42): 602-11.
41. Weddle M e Kokotailo P. Adolescent substance abuse Confidentiality and consent. Pediatri Clin N Am 2002; (49):301-15.

42. Projeto MATCH research group: Matching alcoholism treatments to client heterogeneity: Projeto MATCH posttreatment drinking outcomes. J Stud Alcohol 1997; (58) 729.

43. Wurmser L. Psychoanalytic considerations of the etiology of compulsive drug use. Journal of the American Psychoanalytic Association 1974; (22): 820-43.

44. Dodes $\mathrm{L}$. The psychology of combining dynamic psychotherapy and Alcoholics Anonymous. Bull Menninger Clin 1988; (52): 283-93.

\section{RESUMO}

O uso e o abuso de drogas pelos adolescentes é cada vez mais prevalente e traz desdobramentos sérios nos vários níveis de seu desenvolvimento e na sua família. O presente trabalho aborda as pesquisas no campo epidemiológico sobre fatores de proteção e de risco para o uso de drogas entre os jovens, que demonstram que fracassos tanto pessoais como familiares, além de eventos estressores durante a vida, estão mais associados com o uso de drogas. No entanto, a presença dos pais, a motivação pessoal e o monitoramento do adolescente, estão associados com o não uso. São descritos os principais estudos prospectivos já realizados nessa área. Em seguida, à luz do pensamento de diversos autores como $\mathrm{H}$. Kohut, J. McDougall, H. Rosenfeld, C. Olievenstein, Khantzian, entre outros, apresentam-se teorias psicodinâmicas relacionadas ao problema das adições e sua evolução ao longo dos anos dentro do paradigma psicanalítico. A aplicação das técnicas baseadas nos modelos psicoterápicos de orientação analítica, em dependentes químicos, é também discutida.

Descritores: adolescente, adolescência, psicodinâmica, psicanálise, psicoterapia de orientação analítica, dependência química, abuso e dependência de drogas.

\section{ABSTRACT}

Drug abuse amongst adolescents is becoming increasingly prevalent placing serious consequences in their development and their relationship with family and society. The present paper discloses some epidemiological data on protectors and risk factors for drug abuse in this population demonstrating that personal and family stressors during early life are associated with drug abuse. On the other hand, engaged parents, personal motivation and constant monitoring are associated with avoidance of drugs. Major prospective studies in this field are also reviewed. Psychodynamic theories related to addiction and their evolution through the thoughts of authors such as $H$. Kohut, J. McDougall, H. Rosenfeld, C. Olievenstein, Khantzian, are analyzed. The application of the thecnics based on 
psychoanalytic psychotherapy in chemical dependents is also discussed.

Keywords: Adolescence, adolescent, psychodynamics, psychoanalysis, psychoanalytic psychotherapy, chemical dependency, drug abuse and dependency.

Title: Psychodynamic of the adolescent involved with drugs
Endereço para correspondência:

Felix Kessler

Rua Dona Laura, 354/301

90430-090 - Porto Alegre - RS

Fone: 33328172

E-mail: kessler@ez-poa.com.br

Copyright $\odot$ Revista de Psiquiatria do Rio Grande do Sul - SPRS 